\title{
Educación musical performativa en la formación de intérpretes.
} Un estudio de caso

\section{Performative music education for music students. \\ A case study}

María Fernanda Ramírez Martínez alvta18@hotmail.com Universidad de Granada

Granada, España

ORCID: https://orcid.org/0000-0002-0211-5130

José A. Rodríguez-Quiles kiles@ugr.es

Universidad de Granada

Granada, España

ORCID: https://orcid.org/0000-0003-2490-0540

doi: 10.7203/LEEME.45.16231

Recibido: 03-12-2019 Aceptado: 28-02-2020. Contacto y correspondencia: María Fernanda Ramírez Martínez, Facultad de Ciencias de la Educación Universidad de Granada, Campus de Cartuja s/n, 18071 Granada, España

\section{Resumen}

La educación musical postmoderna viene reclamando la utilización de nuevos modos de conceptualizar los procesos de enseñanzaaprendizaje que hagan más significativos y eficaces los procesos de apropiación y empoderamiento musicales, utilizando para ello herramientas derivadas de la música moderna, del aprendizaje rizomático o del propio contexto social de los participantes. Gracias a esto surgen, entre otras propuestas pedagógicas, el Aprendizaje Musical Informal y la Educación Musical Performativa. La presente investigación es un estudio de caso sobre clases instrumentales colectivas en una institución superior de música de España y de cómo sus prácticas metodológicas se aproximan a las arriba mencionadas, teniendo como base un ambiente de aprendizaje flexible y democrático que repercute de forma significativa en la formación de futuros músicos profesionales.

Palabras clave: educación musical superior; performatividad; educación musical performativa; aprendizaje musical informal.

\begin{abstract}
In postmodern music education there has been a great demand for new ways of conceptualizing teaching-learning processes to make musical appropriation and empowerment more meaningful and effective. This demand calls for the use of tools derived from modern music, from rhizomatic learning as well as those from the participant's own social context. As a result of this demand many educational proposals have arisen, such as the Informal Music Learning and the Performative Music Education This research paper is a case study on collective instrumental classes given in a further education center for music in Spain, made in order to investigate the methodological practices used and to analyse the way in which they are moving towards the practices previously mentioned. It also serves as a means of research to see if these practices have a significant impact on the training of future professional musicians.
\end{abstract}

Key words: higher music education; performativity; performative music education; informal music learning.

@María Fernanda Ramírez Martínez y José A. Rodríguez-Quiles. The content of this article is the sole responsibility of the authors. The Revista Electrónica de LEEME and Universitat de València are not liable for any legal actions that may arise involving the article's content. Revista Electrónica de LEEME - Lista Electrónica Europea de Música en la Educación-. http://ojs.uv.es/index/php/LEEME/index ISSN: 1575-9563. Editores: Universidad de Valencia y Jesús Tejada. Visibilidad de esta revista: SCOPUS, Emerging Sources Citation Index (Clarivate), EBSCO, CINDOC (CSIC), Citefactor, COPAC, Dialnet, DICE (CSIC), DOAJ, e-revistas (CSIC), EBSCO Premier, ERIH+, Gale Cengage Learning, IN-RECS, IRESIE, LATINDEX, MIAR, OCLC Worldcat, RESH, REDIB, RILM Core Journals, SUDOC, ULRICHS. Esta revista es de acceso libre mediante licencia Creative Commons 4.0 CC by. Política de archivado: etiqueta verde SHERPA-ROMEO. 


\section{Introducción}

Los cambios acaecidos en la cultura musical en la época postmoderna han repercutido también en la educación musical. Así, desde hace varias décadas, educadores e investigadores de este ámbito de conocimiento vienen reclamando una ampliación de los géneros musicales a enseñar que no se circunscriba a los de la música académica occidental, con la intención de brindar competencias profesionales en estilos diferentes y alternativos a los de aquella (Woody, 2007). Gran parte de la iniciativa surge con la intención de conectar aquello que se estudia con la realidad y cotidianeidad del alumnado de música cuyos intereses se orientan más hacia la "música popular" o "música moderna" (Mantie, 2013).

El estudio de la música moderna ha supuesto un replanteamiento de las metodologías llevadas a cabo por docentes de diferentes ámbitos educativos y orientadas a diversos tipos de alumnado, brindando múltiples posibilidades y abriendo caminos que posibiliten procesos de aprendizaje más dinámicos y pertinentes que los que se venían empleando, arguyendo razones como la motivación del alumnado (Flores, 2007), el desarrollo de habilidades creativas (Hall, 2014), el aprendizaje de habilidades musicales (Pulman, 2014) o el desarrollo profesional del músico (Jenkins, 2011; Tobias, 2013), entre otras. De este modo, se encuentran diversas aproximaciones pedagógicas que pretenden crear una relación entre el contexto del alumnado y su propio aprendizaje, así como dotarlos de herramientas para que sean ellos mismos los que, guiados por el docente, logren resolver el puzle de conocimientos y competencias requerido para convertirse en músicos profesionales. Atención especial merecen las propuestas orientadas a generar un ambiente de aprendizaje flexible, en el cual se desarrollen conocimientos y habilidades a través de acontecimientos performativos similares a aquellos propios del campo profesional. Es el caso de la Educación Musical Performativa (en adelante, EMP) (RodríguezQuiles, 2017, 2018) y del Aprendizaje Musical Informal (en adelante, AMI) (Green, 2002, 2004, 2006; Jenkins, 2011; Monk, 2017). Ambas propuestas son tomadas en esta investigación como marco teórico de referencia a partir del cual analizar, reflexionar y reconstruir la práctica educativo-musical para futuros intérpretes.

En efecto, en el ámbito de la educación musical superior, estas conceptualizaciones metodológicas pueden ser igualmente empleadas para capacitar al futuro músico-intérprete y brindarle herramientas pedagógicas que puedan optimizar el aprendizaje musical (Feichas, 2010). Sin embargo, no es claro en qué medida son utilizadas en la formación de intérpretes en educación superior. El presente trabajo intenta así, arrojar luz a este campo a partir de las siguientes preguntas de investigación y tomando como objeto de análisis la clase de combo' ${ }^{1}$ :

\footnotetext{
${ }^{1}$ Según la RAE, la palabra combo proviene del inglés, como abreviación de combination. Aquí se entenderá por combo la agrupación o ensemble instrumental y vocal destinado a la interpretación de músicas populares de diferentes estilos (Hard Bop, Latin Jazz, Neo Soul, Salsa...), sin la presencia de un director en escena.
}

@María Fernanda Ramírez Martínez y José A. Rodríguez-Quiles. The content of this article is the sole responsibility of the authors. The Revista Electrónica de LEEME and Universitat de València are not liable for any legal actions that may arise involving the article's content. Revista Electrónica de LEEME - Lista Electrónica Europea de Música en la Educación-. http://ojs.uv.es/index/php/LEEME/index ISSN: 1575-9563. Editores: Universidad de Valencia y Jesús Tejada. Visibilidad de esta revista: SCOPUS, Emerging Sources Citation Index (Clarivate), EBSCO, CINDOC (CSIC), Citefactor, COPAC, Dialnet, DICE (CSIC), DOAJ, e-revistas (CSIC), EBSCO Premier, ERIH+, Gale Cengage Learning, IN-RECS, IRESIE, LATINDEX, MIAR, OCLC Worldcat, RESH, REDIB, RILM Core Journals, SUDOC, ULRICHS. Esta revista es de acceso libre mediante licencia Creative Commons 4.0 CC by. Política de archivado: etiqueta verde SHERPA-ROMEO. 
1. ¿Cómo se desarrollan las prácticas metodológicas llevadas a cabo en la clase de combo de un programa oficial de estudios superiores en música moderna?

2. ¿Qué características de la Educación Musical Performativa presenta y qué efectos educativos provocan en el alumnado?

A partir de estas preguntas iniciales, se persigue los siguientes objetivos:

a) Describir las características de las prácticas metodológicas utilizadas por el profesorado en la clase de combo, así como su contenido performativo.

b) Identificar la pertinencia de las metodologías implementadas y su repercusión en la motivación del alumnado.

c) Identificar en qué medida las prácticas metodológicas llevadas a cabo promueven el empoderamiento del alumnado como futuro intérprete profesional.

\section{Educación Musical Performativa (EMP) y Aprendizaje Musical Informal (AMI)}

La performance musical hace referencia a la representación en vivo por uno o varios intérpretes de una obra musical en un espacio-tiempo determinado y, generalmente, en presencia de una audiencia o público. Por su parte, el concepto performatividad surgió en el ámbito de la Filosofía del Lenguaje de la mano de Austin en 1961(2010), siendo numerosas y diversas las disciplinas que posteriormente lo han venido aplicando exitosamente en sus respectivos campos de estudio (Sociología, Estudios de Género, Teología, Performance Studies, Educación, Artes...).

Kartomi (2014) emplea el concepto performatividad en el ámbito de la Música para hacer referencia a todos aquellos componentes y características que describen y definen aspectos emergentes mientras el músico está llevando a cabo una actuación musical, así como los factores que influyen en el evento y en su preparación previa, refiriéndose a la persona intérprete, a su competencia, a la forma de abordar la performance y su creación, al estilo musical, y otros aspectos como el repertorio, el ensayo o la interacción entre intérpretes de un mismo grupo musical que al final definirá el grado de sincronía y comunicación sobre el escenario. Por su parte, Rodríguez-Quiles -a partir de diferentes trabajos sobre cultura performativa (Bachmann-Medik, 2006; Fischer-Lichte, 2004, 2012; Volbers, 2014) y en lo que ha definido como Educación Musical Performativa (2017, 2018) - focaliza la atención en aquello que acontece en el aula de Música cuando se ponen en juego las denominadas performances didáctico-musicales (PDMs) y cómo estas facilitan la adquisición de conocimientos y habilidades mediante procesos educativos que empoderan al alumnado a partir de procesos autorreferenciales y constituyentes de realidades estético-musicales.

@ María Fernanda Ramírez Martínez y José A. Rodríguez-Quiles. The content of this article is the sole responsibility of the authors. The Revista Electrónica de LEEME and Universitat de València are not liable for any legal actions that may arise involving the article's content. Revista Electrónica de LEEME - Lista Electrónica Europea de Música en la Educación-. http://ojs.uv.es/index/php/LEEME/index ISSN: 1575-9563. Editores: Universidad de Valencia y Jesús Tejada. Visibilidad de esta revista: SCOPUS, Emerging Sources Citation Index (Clarivate), EBSCO, CINDOC (CSIC), Citefactor, COPAC, Dialnet, DICE (CSIC), DOAI, e-revistas (CSIC), EBSCO Premier, ERIH+, Gale Cengage Learning, IN-RECS, IRESIE, LATINDEX, MIAR, OCLC Worldcat, RESH, REDIB, RILM Core Journals, SUDOC, ULRICHS. Esta revista es de acceso libre mediante licencia Creative Commons 4.0 CC by. Política de archivado: etiqueta verde SHERPA-ROMEO. 
Tomando como base estos dos pilares, performance y performatividad musical, la educación musical podría beneficiarse en gran medida a partir de aproximaciones pedagógicas y didácticas centradas no solamente en el estudio de la teoría de la música, la evolución histórica del lenguaje musical, las técnicas de interpretación instrumental, etc., sino en la creación de la performance propiamente dicha como acontecimiento único e irrepetible y de sus consecuencias performativas. Así, se podrá ofrecer una experiencia educativo-musical que, siendo significativa para el alumnado, consiga hacer del hecho musical una experiencia social que trascienda la partitura y los recursos técnicos en su sentido tradicional para transformar el entorno socioeducativo en el que se origina y desarrolla. Es por esto que la EMP presta atención a la experiencia artístico-musical teniendo en cuenta cada aspecto del proceso pedagógico musical y de los aspectos performativos derivados del acontecimiento estético. Esta experiencia educativa se muestra como única e irrepetible en cada contexto, según el flow de la clase. Por tanto, no puede ser planificada y cerrada con antelación en su totalidad, pues buena parte de ella se despliega desde el propio ambiente que genera el acontecimiento como consecuencia de las interacciones en el seno del grupo-clase (docente incluido), estando siempre abierta a las necesidades educativas del momento (Rodríguez-Quiles, 2018).

La EMP viene definida por las siguientes cuatro características principales (RodríguezQuiles, 2017): 1) relación existente entre intencionalidad y emergencia; 2) todos los participantes son co-creadores; 3) es un proceso eminentemente socio-educativo; 4) presenta un alto nivel de componente ético. Así pues, por un lado, la EMP se centra en primera instancia en los eventos que acontecen en clase y en la experiencia social que se desarrolla a través de la praxis musical. En efecto, la atención a los procesos performativos viene tomando tanta fuerza como el estudio de la performance en sí, ya que permite analizar la efectividad y el resultado de la performance desde dentro del evento mismo, estudiando sus características y procesos internos (Davidson, 2014). Considerando el proceso de enseñanza-aprendizaje musical como performance (Pineau, 2005), se hace necesario indagar también en esta línea performativa en el aula. Por otro lado, el AMI (Green, 2013) es otra de las conceptualizaciones pedagógicas que aplica elementos de la EMP, pues propone un ambiente de aprendizaje menos tradicional y más flexible que rescata elementos característicos de la forma no académica en cómo aprenden y han aprendido a lo largo de la historia diferentes músicos de estilos tradicionales, folclóricos, jazz, flamenco o populares; aficionados que adquieren sus habilidades musicales por medio de actividades libres y muchas veces espontáneas, tales como imitar, escuchar, compartir entre amigos o llevar a cabo una performance real, abordando la música que es de su agrado sin seguir un plan estricto ni partiendo de la lecto-escritura musical como condición sine qua non para hacer música.

El aprendizaje informal fuera de un marco académico no precisa de profesorado al uso, planes de estudios, evaluaciones estandarizadas, ni otros elementos predeterminados que deban ser seguidos al pie de la letra por el aprendiz (Vidmar, 2014). Antes bien, se realiza de manera

@ María Fernanda Ramírez Martínez y José A. Rodríguez-Quiles. The content of this article is the sole responsibility of the authors. The Revista Electrónica de LEEME and Universitat de València are not liable for any legal actions that may arise involving the article's content. Revista Electrónica de LEEME - Lista Electrónica Europea de Música en la Educación-. http://ojs.uv.es/index/php/LEEME/index ISSN: 1575-9563. Editores: Universidad de Valencia y Jesús Tejada. Visibilidad de esta revista: SCOPUS, Emerging Sources Citation Index (Clarivate), EBSCO, CINDOC (CSIC), Citefactor, COPAC, Dialnet, DICE (CSIC), DOAJ, e-revistas (CSIC), EBSCO Premier, ERIH+, Gale Cengage Learning, IN-RECS, IRESIE, LATINDEX, MIAR, OCLC Worldcat, RESH, REDIB, RILM Core Journals, SUDOC, ULRICHS. Esta revista es de acceso libre mediante licencia Creative Commons 4.0 CC by. Política de archivado: etiqueta verde SHERPA-ROMEO. 
espontánea, autónoma y libre, dependiendo tanto de la motivación intrínseca del alumnado, como de la extrínseca que el contexto en que tiene lugar pueda ofrecer. De la misma manera, es evidente que la mayor parte de la música que se escucha globalmente a diario es interpretada por músicos que han tenido un aprendizaje informal, al margen de instituciones educativas al uso. Green (2014) sostiene que la educación musical actual se vería beneficiada si tuviera en cuenta las diferentes prácticas y características del aprendizaje informal y los extrapolase a la educación formal con el objetivo de crear un ambiente de aprendizaje más efectivo y significativo en cuanto se presenta, por lo general, cómodo y sin la presión por obtener una calificación numérica (Mok, 2017). El alumnado es motivado por el simple hecho de vivir la experiencia musical y lograr el objetivo planteado por el guía, lo cual, -aplicado a las prácticas institucionalizadas-, requiere un cambio de paradigma tanto para el docente como para el discente (Rodríguez-Quiles, 2018).

\section{Método}

Se ha llevado a cabo una investigación de carácter cualitativo, en cuanto se pretende descubrir y describir los fenómenos pedagógicos implícitos en la práctica analizada, así como también proveer una comprensión de las actividades educativas mediante una perspectiva descriptiva y analítica. En concreto, se trata de un estudio instrumental de caso (Stake, 1998) cuya finalidad es entender los procesos llevados a cabo por un grupo de personas sin centrarse en la comprensión de los sujetos, sino en los efectos de sus acciones, con el objetivo de obtener mayor entendimiento de la temática en cuestión.

La investigación se ha llevado a cabo en la Escuela Superior de Música de Cataluña, con la autorización expresa del jefe de departamento correspondiente, así como de la coordinadora de investigación de la institución. Profesorado y alumnado aceptaron, igualmente por escrito, colaborar para esta investigación, permitiendo ser entrevistados y grabados en soporte audiovisual. En total, se grabaron 35 horas de clase, divididas en seis sesiones semanales de noventa minutos correspondientes a cuatro combos diferentes cuyo objetivo es la interpretación de obras de un estilo o artista determinado, tal como se indicará más adelante. Además, se realizaron ocho entrevistas semiestructuradas durante los meses de octubre y noviembre de 2017.

Los datos obtenidos por medio de esta investigación fueron analizados a partir de un sistema de categorías, subcategorías y códigos de tipo emergente. Para realizar una categorización apriorística con ayuda del programa NVivo 12, se tuvieron en cuenta aspectos propios de la investigación en EMP (Rodríguez-Quiles, 2017), así como conceptos referentes a la investigación en Performatividad Musical (Kartomi, 2014) y en Aprendizaje Informal (Green, 2013).

@María Fernanda Ramírez Martínez y José A. Rodríguez-Quiles. The content of this article is the sole responsibility of the authors. The Revista Electrónica de LEEME and Universitat de València are not liable for any legal actions that may arise involving the article's content. Revista Electrónica de LEEME - Lista Electrónica Europea de Música en la Educación-. http://ojs.uv.es/index/php/LEEME/index ISSN: 1575-9563. Editores: Universidad de Valencia y Jesús Tejada. Visibilidad de esta revista: SCOPUS, Emerging Sources Citation Index (Clarivate), EBSCO, CINDOC (CSIC), Citefactor, COPAC, Dialnet, DICE (CSIC), DOAJ, e-revistas (CSIC), EBSCO Premier, ERIH+, Gale Cengage Learning, IN-RECS, IRESIE, LATINDEX, MIAR, OCLC Worldcat, RESH, REDIB, RILM Core Journals, SUDOC, ULRICHS. Esta revista es de acceso libre mediante licencia Creative Commons 4.0 CC by. Política de archivado: etiqueta verde SHERPA-ROMEO. 


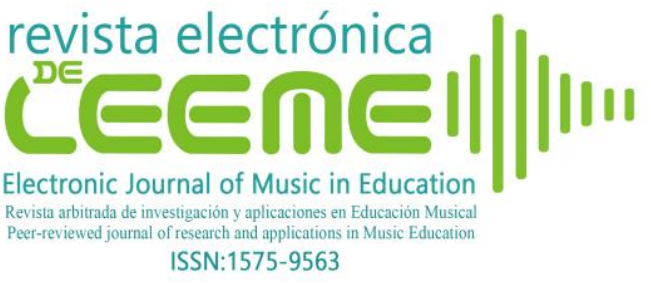

María Fernanda Ramírez Martínez y José A. Rodríguez-Quiles Educación musical performativa en la formación de intérpretes. Un estudio de caso (Revista Electrónica de LEEME) Número 45, pp. 17-34 https://ojs.uv.es/index.php/LEEME/index DOI: 10.7203/LEEME.45.1623

Las clases de combo analizadas forman parte del programa de estudios conducente al Título Superior en Música - Interpretación de Jazz y Música Moderna y se desarrollaron como se explica a continuación, siguiendo el plan de estudios establecido: desde el primer año de la titulación, el alumnado participa en diferentes clases de combo con sesiones semanales de 90 minutos a lo largo de un semestre; en primero y segundo año, se ofrecen combos prediseñados y preestablecidos por el equipo docente, entre los cuales el estudiante debe escoger dos por año, estando enfocados a géneros y estilos que, a juicio del profesorado, forman parte de la instrucción básica de todo intérprete de Jazz y Música Moderna. Algunos de estos combos son: combo de Miles David, combo de Hard Bop, combo de Latin Jazz y combo de Neo Soul. A partir del tercer año, el alumnado puede proponer el combo a cursar durante el semestre, especificando el estilo musical deseado, la agrupación de estudiantes que lo formará y el profesorado que lo guiará. Previo a su aceptación o no, la propuesta es estudiada por el departamento competente, así como por el docente sugerido. De los cuatro combos analizados aquí, dos forman parte del programa de estudios y se ofrecen cada año, mientras los otros dos fueron propuestos por el alumnado.

\section{Resultados}

La siguiente tabla muestra la categorización realizada para el análisis de las grabaciones de vídeo de las clases de combo y las entrevistas a profesorado y alumnado.

Tabla 1. Categorías analizadas

\begin{tabular}{|c|c|c|}
\hline CATEGORÍA & CONCEPTOS & BREVE DESCRIPCIÓN \\
\hline Horizontalidad & $\begin{array}{ll}\text { - } & \text { Horizontalidad (EMP) } \\
\text { - } & \text { Aprendizaje informal en clase } \\
(\mathrm{AMI}) & \end{array}$ & $\begin{array}{l}\text { Relaciones } \quad \text { profesorado- } \\
\text { alumnado }\end{array}$ \\
\hline Espacialidad & - $\quad$ Espacialidad (EMP) & $\begin{array}{l}\text { Uso performativo de los } \\
\text { espacios como parte de los } \\
\text { procesos } \\
\text { enseñanza/aprendizaje }\end{array}$ \\
\hline Corporalidad & - $\quad$ Corporalidad (EMP) & $\begin{array}{l}\text { Uso consciente del cuerpo y } \\
\text { su influencia en los procesos } \\
\text { de enseñanza/aprendizaje }\end{array}$ \\
\hline Repertorio Musical & $\begin{array}{ll}\text { - } & \text { Heterogeneidad (EMP) } \\
\text { - } & \text { Enculturación (músicos populares) } \\
\text { - } & \text { Repertorio escogido por alumnado } \\
\text { (AMI) }\end{array}$ & $\begin{array}{l}\text { Repertorio utilizado, estilos } \\
\text { musicales, apertura a nuevos } \\
\text { estilos o nuevas formas } \\
\text { musicales }\end{array}$ \\
\hline Aprendizaje colaborativo & $\begin{array}{l}\text { - } \quad \begin{array}{l}\text { Tocar con conocidos (músicos } \\
\text { populares) }\end{array} \\
\end{array}$ & $\begin{array}{l}\text { Trabajo grupal, relación entre } \\
\text { estudiantes y cómo influye en }\end{array}$ \\
\hline
\end{tabular}

@María Fernanda Ramírez Martínez y José A. Rodríguez-Quiles. The content of this article is the sole responsibility of the authors. The Revista Electrónica de LEEME and Universitat de València are not liable for any legal actions that may arise involving the article's content. Revista Electrónica de LEEME - Lista Electrónica Europea de Música en la Educación-. http://ojs.uv.es/index/php/LEEME/index ISSN: 1575-9563. Editores: Universidad de Valencia y Jesús Tejada. Visibilidad de esta revista: SCOPUS, Emerging Sources Citation Index (Clarivate), EBSCO, CINDOC (CSIC), Citefactor, COPAC, Dialnet, DICE (CSIC), DOAJ, e-revistas (CSIC), EBSCO Premier, ERIH+, Gale Cengage Learning, IN-RECS, IRESIE, LATINDEX, MIAR, OCLC Worldcat, RESH, REDIB, RILM Core Journals, SUDOC, ULRICHS. Esta revista es de acceso libre mediante licencia Creative Commons 4.0 CC by. Política de archivado: etiqueta verde SHERPA-ROMEO. 
Tabla 1. Categorías analizadas

\begin{tabular}{|c|c|c|}
\hline & - $\quad$ Aprendizaje cooperativo (AMI) & el desarrollo de la clase \\
\hline Aprendizaje emergente & $\begin{array}{ll}- & \text { Cartografia (EMP) } \\
\text { - } & \text { Aprendizaje emergente y holístico } \\
\text { - } & \text { Aprendizaje significativo }\end{array}$ & $\begin{array}{l}\text { Aprendizaje que se crea sin } \\
\text { planificación expresa pero } \\
\text { que resulta contextualmente } \\
\text { significativo }\end{array}$ \\
\hline Esteticidad & - $\quad$ Esteticidad (EMP) & $\begin{array}{l}\text { Atención a los detalles } \\
\text { estéticos de performance }\end{array}$ \\
\hline Ética & - $\quad$ Eticidad (EMP) & $\begin{array}{lrr}\begin{array}{l}\text { Atención } \\
\text { componente }\end{array} & \begin{array}{r}\text { expresa } \\
\text { ético }\end{array} & \text { al } \\
\text { procesos pedagógicos } & \end{array}$ \\
\hline Materialidad & - $\quad$ Materialidad (EMP) & $\begin{array}{l}\text { Uso convencional } 0 \text { no } \\
\text { convencional del material } \\
\text { sonoro }\end{array}$ \\
\hline Disfrute & $\begin{array}{ll}\text { - } & \text { Disfrute (Músicos populares) } \\
\text { - } & \text { Autoestima (músicos populares) } \\
\text { - } & \text { Motivación }\end{array}$ & $\begin{array}{l}\text { Identificación del alumnado } \\
\text { con los procesos educativos }\end{array}$ \\
\hline
\end{tabular}

Fuente: elaboración propia

Por motivos de espacio y ya que la investigación puede considerarse un estudio en desarrollo, presentamos únicamente el análisis de aquellas categorías que han resultado especialmente significativas, a partir de las entrevistas realizadas a profesorado y alumnado.

\section{a) Horizontalidad}

Se refiere a la relación que existe entre alumnado y profesorado que, desde la perspectiva de la EMP y el AMI, se basa en un trato horizontal en donde el alumnado adquiere mayor autonomía y el docente se convierte en guía o facilitador de los procesos de aprendizaje sin ser el único "dueño" del conocimiento ni de las decisiones tomadas dentro de la clase (Feichas, 2010).

Como ya se ha mencionado, algunas clases de combo son propuestas por el alumnado, quienes preparan un documento en donde explican el estilo o artista en el que se basará el combo, el profesorado propuesto para impartir la clase y los integrantes de la agrupación instrumental. Esta posibilidad dentro de la institución, en un programa de estudios superiores, supone una apuesta por un trato horizontal en las relaciones profesorado-alumnado, que incluso va más allá, incluyendo a la institución propiamente dicha, desde el momento en que el alumnado toma parte en decisiones organizativas y pedagógicas de su titulación. Esto no es común en la mayor parte de las instituciones educativas del país y muy bien podría aplicarse a otras especialidades de los Conservatorios de Música del resto del territorio nacional, tanto Superiores como Profesionales.

De igual manera, se observa cómo en algunos casos el profesorado trata de hacer partícipe al alumnado en el desarrollo de la clase y en la toma de decisiones frente a aspectos

@ María Fernanda Ramírez Martínez y José A. Rodríguez-Quiles. The content of this article is the sole responsibility of the authors. The Revista Electrónica de LEEME and Universitat de València are not liable for any legal actions that may arise involving the article's content. Revista Electrónica de LEEME - Lista Electrónica Europea de Música en la Educación-. http://ojs.uv.es/index/php/LEEME/index ISSN: 1575-9563. Editores: Universidad de Valencia y Jesús Tejada. Visibilidad de esta revista: SCOPUS, Emerging Sources Citation Index (Clarivate), EBSCO, CINDOC (CSIC), Citefactor, COPAC, Dialnet, DICE (CSIC), DOAJ, e-revistas (CSIC), EBSCO Premier, ERIH+, Gale Cengage Learning, IN-RECS, IRESIE, LATINDEX, MIAR, OCLC Worldcat, RESH, REDIB, RILM Core Journals, SUDOC, ULRICHS. Esta revista es de acceso libre mediante licencia Creative Commons 4.0 CC by. Política de archivado: etiqueta verde SHERPA-ROMEO. 
como repertorio, arreglos musicales o detalles interpretativos, diferenciándose del sistema tradicional de enseñanza, en el cual docente y discente tienen roles definidos y jerárquicamente establecidos:

\begin{abstract}
Mi participación siempre funciona [en el sentido] de filtrar cosas: ellos proponen una serie de cosas, y yo, de alguna manera, lo que hago es hacerles ver que hay que quedarse con la opción más válida, -sonora y estéticamente-, dentro del género. Siempre intento ser muy crítico con ellos. Cuando hay algo que realmente lo prueban y no funciona, es mejor ir a buscar otra cosa, pero [muy a menudo] les dejo decidir cuáles son las cosas que funcionan [...]. Tienen que ser críticos de su propio trabajo, porque el día de mañana lo tendrán que hacer por ellos mismos. (Entrevista docente $\mathrm{W}$ )
\end{abstract}

Como subraya Green (2013), los músicos populares que han tenido éxito en adquirir habilidades y convertirse en músicos profesionales practican de manera flexible sin la instrucción de un maestro incuestionable y 'sabelotodo' que les enseña la única manera correcta de tocar un instrumento. Esta práctica informal de aprendizaje brinda experiencias significativas para el alumnado y podría ser tenida en cuenta en la educación formal con el fin de facilitar el empoderamiento del alumnado gracias a un rol más cercano, flexible y enriquecedor del docente desde la perspectiva de una EMP, lo que nunca supone -obviamente- subestimar la misión del profesor.

\title{
b) Aprendizaje colaborativo
}

Es interesante reseñar cómo, en la mayoría de los casos, el alumnado de los combos analizados se conocía antes de comenzar la asignatura. Al haber asistido conjuntamente a clases de otras materias en cursos anteriores, han tenido la posibilidad de tocar juntos, cuando ni siquiera mantienen relaciones de amistad fuera del centro. Este hecho afecta directamente a la forma en que interactúan entre ellos en clase y al desarrollo de las actividades de aula. Refiriéndose a los músicos populares, Green (2013) apunta como una de las características del aprendizaje informal el hecho de que generalmente se aprende tocando con amigos en un entorno familiar y de confianza, lo cual facilita un ambiente adecuado en el que todos participan y logran un trabajo en equipo desde la confianza, tolerancia y amistad:

Es como tocar en familia. Todos nos conocemos y podemos fallar... Si tenemos un día malo o un día bueno, si estoy enferma... Hay esa confianza y el ambiente amigable de que todos estamos allí para aprender. (Entrevista alumna N)

Respecto a los combos propuestos por el alumnado, declaran haber escogido en primera instancia atendiendo a la relación de amistad que les unía antes de cursar la asignatura. En las ocasiones en que esto no fue posible, escogieron a algún otro estudiante por su buen nivel instrumental o por la buena impresión que le había causado a nivel personal. En cualquier caso, se evidencia que el alumnado se siente cómodo tocando con amigos o conocidos y que este aspecto potencia las posibilidades educativas de la clase, promoviendo la obtención de

@María Fernanda Ramírez Martínez y José A. Rodríguez-Quiles. The content of this article is the sole responsibility of the authors. The Revista Electrónica de LEEME and Universitat de València are not liable for any legal actions that may arise involving the article's content. Revista Electrónica de LEEME - Lista Electrónica Europea de Música en la Educación-. http://ojs.uv.es/index/php/LEEME/index ISSN: 1575-9563. Editores: Universidad de Valencia y Jesús Tejada. Visibilidad de esta revista: SCOPUS, Emerging Sources Citation Index (Clarivate), EBSCO, CINDOC (CSIC), Citefactor, COPAC, Dialnet, DICE (CSIC), DOAJ, e-revistas (CSIC), EBSCO Premier, ERIH+, Gale Cengage Learning, IN-RECS, IRESIE, LATINDEX, MIAR, OCLC Worldcat, RESH, REDIB, RILM Core Journals, SUDOC, ULRICHS. Esta revista es de acceso libre mediante licencia Creative Commons 4.0 CC by. Política de archivado: etiqueta verde SHERPA-ROMEO. 
resultados y desarrollando competencias profesionales de carácter grupal (Green, 2013; Reid y Duke, 2015).

De igual manera, el profesorado subraya la importancia del trabajo en equipo para el adecuado desarrollo de las clases, desde el momento en que el combo se basa en la creación colectiva y el aprendizaje cooperativo (Abrahams, Rowland y Kohler, 2012; Egger, 2019; Espinosa, 2019; Harrison, Lebler, Carey, Hitchcock y O’Bryan, 2013; Whitener, 2016):

En un equipo en donde hay un rol de trabajo, es mucho más fácil conseguir resultados con un nivel medio de conocimientos que en un equipo en el que hay un nivel óptimo de conocimientos, pero el rol de trabajo es negativo y hay malos entendimientos... (Entrevista docente $\mathrm{W}$ )

De igual manera, las relaciones interpersonales que se van forjando entre el alumnado, junto a la personalidad de cada uno y su capacidad para trabajar en equipo, influyen directamente sobre la performance:

Los metales son una familia que al final funcionan como una sola persona. [...]. Cuando 'hablan' como solistas independientes tienes más problemas que cuando entre todos ya hay una dinámica $[y]$ se sabe que hay que conseguir un solo resultado. (Entrevista docente W)

\section{c) Repertorio musical}

Como se ha dicho, cada combo se enfoca hacia la apropiación grupal del lenguaje musical de un estilo específico. La variedad de estilos estudiados a lo largo de la titulación permite que el alumnado pueda implicarse en diferentes tipos de música moderna sin determinar el grado de importancia, valor, estética o relaciones de subordinación entre diferentes músicas, sino más bien promoviendo la heterogeneidad propia de la música en sentido amplio. Se evidencia que la inclusión de las músicas populares en la educación musical, como herramienta pedagógica, trae consigo la aparición de experiencias informales dentro de la educación reglada; experiencias que promueven a su vez la autenticidad y el aprendizaje significativo en el alumnado (Karlsen, 2010).

Rodríguez-Quiles (2018) explica la importancia de la categoría heterogeneidad como herramienta pedagógica para descentralizar el proceso de enseñanza/aprendizaje y permitir experiencias musicales creadas desde contextos emergentes y rizomáticos ${ }^{2}$, sin propuestas cerradas que puedan coartar, minimizar o incluso prohibir el uso de algún tipo de música. De igual manera, para Green (2013) el tipo de música que se decide abordar es un aspecto importante en el AMI, ya que los músicos populares aprenden informalmente a través de fenómenos de enculturación, refiriéndose al aprendizaje de estilos musicales que son conocidos

\footnotetext{
${ }^{2} \mathrm{El}$ aprendizaje rizomático es aquel en el que el conocimiento es construido por cada persona autoconsciente en aras
} de adaptarse a las condiciones ambientales.

@María Fernanda Ramírez Martínez y José A. Rodríguez-Quiles. The content of this article is the sole responsibility of the authors. The Revista Electrónica de LEEME and Universitat de València are not liable for any legal actions that may arise involving the article's content. Revista Electrónica de LEEME - Lista Electrónica Europea de Música en la Educación-. http://ojs.uv.es/index/php/LEEME/index ISSN: 1575-9563. Editores: Universidad de Valencia y Jesús Tejada. Visibilidad de esta revista: SCOPUS, Emerging Sources Citation Index (Clarivate), EBSCO, CINDOC (CSIC), Citefactor, COPAC, Dialnet, DICE (CSIC), DOAJ, e-revistas (CSIC), EBSCO Premier, ERIH+, Gale Cengage Learning, IN-RECS, IRESIE, LATINDEX, MIAR, OCLC Worldcat, RESH, REDIB, RILM Core Journals, SUDOC, ULRICHS. Esta revista es de acceso libre mediante licencia Creative Commons 4.0 CC by. Política de archivado: etiqueta verde SHERPA-ROMEO. 


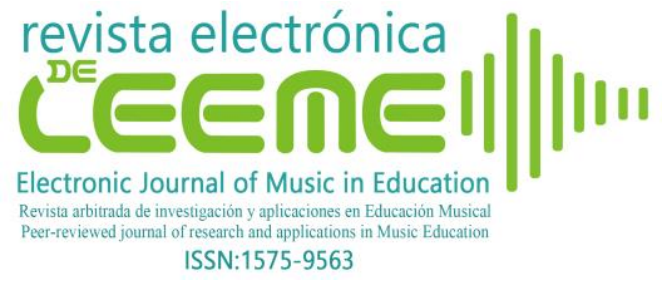

María Fernanda Ramírez Martínez y José A. Rodríguez-Quiles Educación musical performativa en la formación de intérpretes. Un estudio de caso (Revista Electrónica de LEEME) Número 45, pp. 17-34 https://ojs.uv.es/index.php/LEEME/index DOI: $10.7203 /$ LEEME.45.16231

y de uso cotidiano en su contexto familiar o social, esto es, desde la música que para ellos es significativa (Mok, 2017). En este estudio de caso, el alumnado escoge el tipo de música que quiere aprender y de los combos analizados se desprende que esta característica es de gran importancia para el ambiente de aprendizaje y para su motivación, así como para la apropiación del estilo musical deseado junto con el lenguaje que le es propio y lo define:

Antes había tocado los típicos temas de Jobim, pero está "guay" conocer temas más "raros"... Yo propuse unos temas y el profe propuso otros... Tengo muchas expectativas con [la clase de] combo. Me encanta [el] estilo [musical que tocamos]. (Entrevista alumna J)

Es una cosa muy buena que ofrece [el centro]: gran variedad de combos de muchos estilos, [lo que] te permite tocar muchos géneros que quizás no harías de otro modo y eso está bien. Algunos profes dicen que tienes que salir de aquí sabiendo tocar todos los estilos, que tengas las herramientas para poder [abordar] todos los estilos que te puedas encontrar. Los combos son una herramienta fantástica. (Entrevista alumno A)

\section{d) Espacialidad y Corporalidad}

Las clases de combo tienen lugar en aulas dotadas de una infraestructura adecuada. Alumnado y profesorado se ubican libremente en el espacio. Como se observa en la Figura 1, la opción preferida es el círculo, lo que facilita la comunicación entre los miembros, enriqueciendo con ello el trabajo cooperativo.

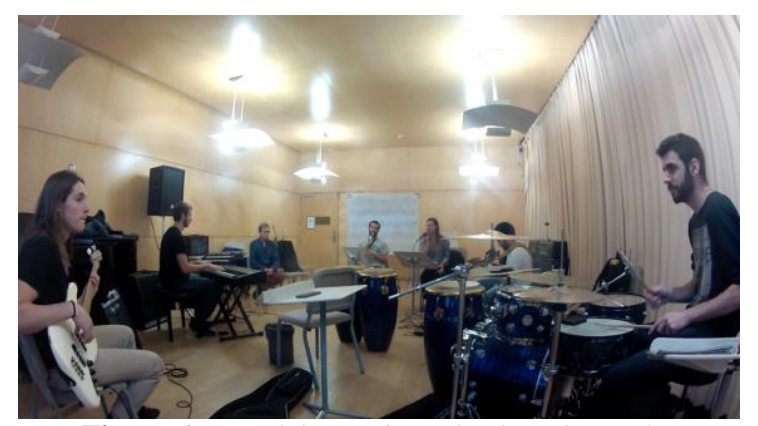

Figura 1. Uso del espacio en la clase de combo Fuente: elaboración propia

Del análisis del trabajo de campo, se deduce que la categoría espacialidad no ha resultado ser un elemento especialmente atendido en el contexto pedagógico de las clases. En EMP, por el contrario, se busca expresamente romper con los usos estándares de los espacios a fin de generar experiencias significativas que puedan empoderar al alumnado. Entonces, no se trataría únicamente de buscar ese uso performativo y no convencional del espacio como herramienta pedagógica alternativa, sino de crear consciencia del espacio en sí y de la manera en que este puede enriquecer la expresión musical brindando experiencias performativas de gran valor en la actividad docente, primero, y en la profesional, después (Rodríguez-Quiles, 2017).

@María Fernanda Ramírez Martínez y José A. Rodríguez-Quiles. The content of this article is the sole responsibility of the authors. The Revista Electrónica de LEEME and Universitat de València are not liable for any legal actions that may arise involving the article's content. Revista Electrónica de LEEME - Lista Electrónica Europea de Música en la Educación-. http://ojs.uv.es/index/php/LEEME/index ISSN: 1575-9563. Editores: Universidad de Valencia y Jesús Tejada. Visibilidad de esta revista: SCOPUS, Emerging Sources Citation Index (Clarivate), EBSCO, CINDOC (CSIC), Citefactor, COPAC, Dialnet, DICE (CSIC), DOAJ, e-revistas (CSIC), EBSCO Premier, ERIH+, Gale Cengage Learning, IN-RECS, IRESIE, LATINDEX, MIAR, OCLC Worldcat, RESH, REDIB, RILM Core Journals, SUDOC, ULRICHS. Esta revista es de acceso libre mediante licencia Creative Commons 4.0 CC by. Política de archivado: etiqueta verde SHERPA-ROMEO. 


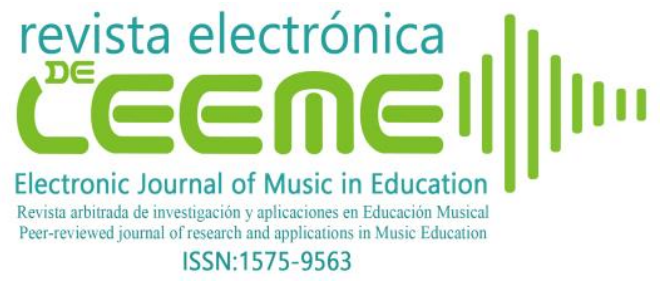

María Fernanda Ramírez Martínez y José A. Rodríguez-Quiles Educación musical performativa en la formación de intérpretes. Un estudio de caso (Revista Electrónica de LEEME) Número 45, pp. 17-34 https://ojs.uv.es/index.php/LEEME/index DOI: 10.7203/LEEME.45.1623

Además del aula en donde se desarrollan las sesiones de clase, el salón de actos se configura como el otro espacio tradicionalmente utilizado por docentes y reservado para la 'audición final' (Figura 2). A diferencia de las clases, en esta audición final, el alumnado se ubica en el escenario de cara al público, como si de un concierto real se tratara, mientras el docente ocupa alguna de las butacas de la sala. Conviene señalar que en ningún momento previo a esta prueba final el alumnado ha hecho uso de dicho espacio, lo que supone una experiencia, si no estrictamente nueva para algunos de ellos, sí para el ensemble en su conjunto. Si bien el profesorado arguye que uno de los objetivos de la clase es el de preparar al alumnado para la profesión musical, no se ahonda sin embargo en los detalles escénicos y en los diferentes aspectos que se ponen en juego en el momento de presentar una performance ante el público: prueba de sonido, ubicación en tarima, miedo escénico, empatía con los oyentes/espectadores... Así como tampoco se desprende del material analizado un uso consciente del cuerpo en escena, dirigiéndose el foco de atención en clase más hacia aspectos de tipo técnico-musical.

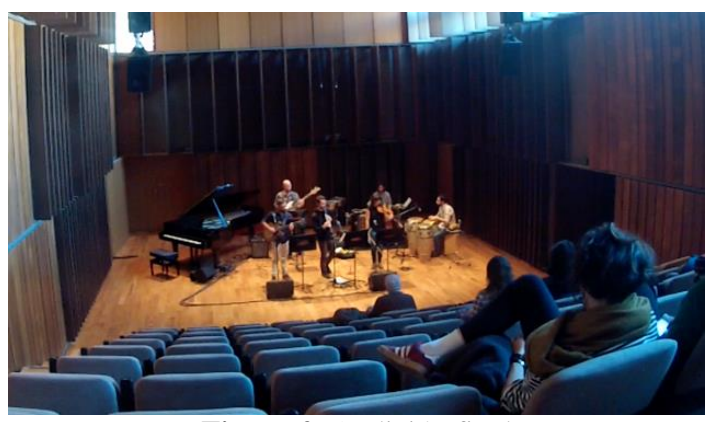

Figura 2. Audición final

Fuente: elaboración propia

\section{e) Aprendizaje emergente}

La Unvorhersehbarkeit o impredecibilidad como parte consustancial a los actos performativos (Fischer-Lichte, 2012) constituye sin duda una de las categorías más interesantes entre las estudiadas en Artes Escénicas y que muy bien se pueden considerar en los procesos de enseñanza-aprendizaje, en general, y en educación musical, en particular. En efecto, como todo docente ha experimentado, la relación entre la planificación de una clase y la realidad derivada de su implementación varía dependiendo del grupo, del contexto, de las circunstancias, entre otros factores. Así, del análisis de las entrevistas se desprende que el propio alumnado es consciente de la importancia de ese aprendizaje que emerge del devenir mismo de la clase de manera no lineal ni preconcebida. Así, frente a la pregunta ‘¿Crees que has conseguido lo que esperabas?' obtenemos respuestas del tipo:

Sí, mucho. Ahora estoy cantando otras canciones en otras asignaturas y me doy cuenta de todo lo que he absorbido [y] que no tenía. (Entrevista alumno E)

@ María Fernanda Ramírez Martínez y José A. Rodríguez-Quiles. The content of this article is the sole responsibility of the authors. The Revista Electrónica de LEEME and Universitat de València are not liable for any legal actions that may arise involving the article's content. Revista Electrónica de LEEME - Lista Electrónica Europea de Música en la Educación-. http://ojs.uv.es/index/php/LEEME/index ISSN: 1575-9563. Editores: Universidad de Valencia y Jesús Tejada. Visibilidad de esta revista: SCOPUS, Emerging Sources Citation Index (Clarivate), EBSCO, CINDOC (CSIC), Citefactor, COPAC, Dialnet, DICE (CSIC), DOAJ, e-revistas (CSIC), EBSCO Premier, ERIH+, Gale Cengage Learning, IN-RECS, IRESIE, LATINDEX, MIAR, OCLC Worldcat, RESH, REDIB, RILM Core Journals, SUDOC, ULRICHS. Esta revista es de acceso libre mediante licencia Creative Commons 4.0 CC by. Política de archivado: etiqueta verde SHERPA-ROMEO. 


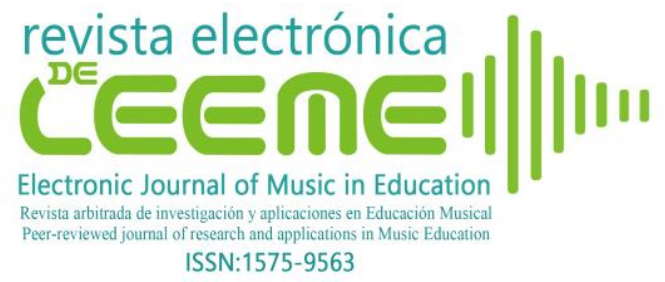

María Fernanda Ramírez Martínez y José A. Rodríguez-Quiles Educación musical performativa en la formación de intérpretes. Un estudio de caso (Revista Electrónica de LEEME) Número 45, pp. 17-34 https://ojs.uv.es/index.php/LEEME/index DOI: 10.7203/LEEME.45.1623

Se aprende versatilidad, a trabajar con poco tiempo, capacidad de reacción, trabajar con gente diferente y saber encajar lo mejor posible. (Entrevista alumno L)

Tengo un grupo [en donde] hago de director musical y todo lo que hago es lo que veo que los profes hacen y lo que he vivido en el combo. (Entrevista alumno $\mathrm{S}$ )

Como explica Green (2013), los músicos populares aprenden de manera informal, -en el marco de una perspectiva performativa-, utilizando como herramienta pedagógica la experiencia de una performance que integra diferentes habilidades, como la escucha y la interpretación. Los conocimientos y habilidades se adquieren así individual y grupalmente, como quien va transitando por un mapa sin un orden o secuencia preestablecida, tomando decisiones de manera emergente. Rodríguez-Quiles (2018), en alusión a Deleuze y Guattari (1980), hace referencia a lo anterior con el término cartografía, una característica del aprendizaje rizomático aplicada a la EMP, en la cual la adquisición de conocimientos se compara con un mapa sobre el que alumnado y profesorado van transitando, explorando y tomando unas rutas u otras dependiendo de los contextos emergentes, así como de las experiencias y relaciones sociales y artísticas que puedan surgir dentro (y fuera) del aula.

\section{f) Eticidad}

El profesorado tiene la responsabilidad de cuidar los aspectos éticos como categoría esencial durante el proceso de enseñanza/aprendizaje (Rodríguez-Quiles, 2017), pues en EMP la horizontalidad y la flexibilidad en la expresión individual y en la toma de decisiones grupales, hacen que las relaciones adquieran un carácter político, lo que resulta evidente cuando algún integrante trata, por ejemplo, de imponer sobre los demás una idea o gusto particular. Uno de los presupuestos esenciales de la EMP consiste en alcanzar un equilibrio entre los elementos artísticos, sociales y políticos para, de esta manera, orientar al alumnado no solo hacia la adquisición de habilidades musicales y profesionales, sino igualmente hacia comportamientos y actitudes moralmente aceptables que le inviten a tomar conciencia de su responsabilidad ante los procesos y los resultados, consecuencia todo ello de un óptimo trabajo colectivo y social.

Se ha comprobado que docentes y discentes son conscientes del componente ético que debe estar en la base del proceso de enseñanza/aprendizaje (Locke, 2008; Elliot, 2014; Nichols, 2016; Richerme, 2017). Al tratarse de una clase grupal en donde la comunicación y las relaciones personales coinstituyen una herramienta de trabajo orientada hacia el cumplimiento de los objetivos, la interacción entre personas repercute directamente sobre el desarrollo y buen resultado de los procesos educativos puestos en juego. El profesorado coincide en otorgar gran importancia al respeto por las propuestas del alumnado y por el trabajo grupal, cuestión ésta que no pasa desapercibida para el alumnado.

Después del combo noté un cambio abismal en cómo yo llevaba las cosas en mi banda: ser mucho más efectivo, saber cómo comunicarte con el resto de los músicos, cómo dirigirlo,

@María Fernanda Ramírez Martínez y José A. Rodríguez-Quiles. The content of this article is the sole responsibility of the authors. The Revista Electrónica de LEEME and Universitat de València are not liable for any legal actions that may arise involving the article's content. Revista Electrónica de LEEME - Lista Electrónica Europea de Música en la Educación-. http://ojs.uv.es/index/php/LEEME/index ISSN: 1575-9563. Editores: Universidad de Valencia y Jesús Tejada. Visibilidad de esta revista: SCOPUS, Emerging Sources Citation Index (Clarivate), EBSCO, CINDOC (CSIC), Citefactor, COPAC, Dialnet, DICE (CSIC), DOAJ, e-revistas (CSIC), EBSCO Premier, ERIH+, Gale Cengage Learning, IN-RECS, IRESIE, LATINDEX, MIAR, OCLC Worldcat, RESH, REDIB, RILM Core Journals, SUDOC, ULRICHS. Esta revista es de acceso libre mediante licencia Creative Commons 4.0 CC by. Política de archivado: etiqueta verde SHERPA-ROMEO. 
respetar mucho más, saber escuchar no solamente a la hora de hablar, sino a la hora de tocar también. A mí me cambió bastante la vida. (Entrevista Alumna N)

\section{g) Esteticidad y disfrute}

Al ser preguntado por los objetivos de la asignatura de combo, el profesorado señala el aspecto estético como objetivo principal, entendido como la apropiación de un estilo específico, siendo capaz de interpretarlo con el resultado más bello posible (Duke, 2006; Hargreaves, 2012; Thakar, 2015). Por otra parte, la motivación y disfrute del alumno aumenta cuando este se enfrenta a músicas significativas para él en ambientes de aprendizaje más informales que exclusivamente formales (Green, 2008; Mok, 2017, 2018).

De igual manera, al abordar una metodología que parte de actividades y tareas próximas a la futura realidad laboral del alumnado y no de pequeños ejercicios de clase descontextualizados, se consigue una mayor motivación, tanto individual como grupal, puesto que los resultados estéticos que se van obteniendo a lo largo del proceso resultarán palpables. Esto, unido a la autonomía que se concede al alumnado como consecuencia de la horizontalidad de los procesos educativos, le permite disfrutar de un ambiente de aprendizaje flexible y cómodo, haciendo que las sesiones -pese al esfuerzo y entrega que conllevan- se asemejen más a momentos de diversión y esparcimiento.

\section{Conclusiones y discusión}

La observación y el análisis de las clases de combo de un programa de estudios superiores de música desde el prisma del Aprendizaje Informal y de la Educación Musical Performativa arrojan conclusiones interesantes sobre innovación en educación musical que merece la pena tener en cuenta y que pasamos a resumir.

Dando respuesta a las preguntas de investigación acerca de las características generales y performativas de las prácticas metodológicas observadas y los efectos educativos sobre el alumnado, se comprueba que la asignatura de combo ofrece al docente gran flexibilidad tanto en la planificación como en el desarrollo de las clases. Por este motivo, los grupos suelen diferenciarse unos de otros, dependiendo del docente que los imparta y de su metodología propia, basada en primera instancia en su criterio y experiencia personales. Aun así, se observa que el profesorado se inclina más por una educación horizontal que facilita al alumnado apropiarse de su singular proceso de aprendizaje (Feichas, 2010), sintiéndose protagonista del desarrollo de la materia en los sucesivos semestres que configuran la titulación. Evidencias de esto se han encontrado en la participación del alumnado en la toma de decisiones y en cómo interpreta su instrumento en el contexto de un ensemble.

@María Fernanda Ramírez Martínez y José A. Rodríguez-Quiles. The content of this article is the sole responsibility of the authors. The Revista Electrónica de LEEME and Universitat de València are not liable for any legal actions that may arise involving the article's content. Revista Electrónica de LEEME - Lista Electrónica Europea de Música en la Educación-. http://ojs.uv.es/index/php/LEEME/index ISSN: 1575-9563. Editores: Universidad de Valencia y Jesús Tejada. Visibilidad de esta revista: SCOPUS, Emerging Sources Citation Index (Clarivate), EBSCO, CINDOC (CSIC), Citefactor, COPAC, Dialnet, DICE (CSIC), DOAJ, e-revistas (CSIC), EBSCO Premier, ERIH+, Gale Cengage Learning, IN-RECS, IRESIE, LATINDEX, MIAR, OCLC Worldcat, RESH, REDIB, RILM Core Journals, SUDOC, ULRICHS. Esta revista es de acceso libre mediante licencia Creative Commons 4.0 CC by. Política de archivado: etiqueta verde SHERPA-ROMEO. 
Por otra parte, este grado de flexibilidad facilita el desarrollo progresivo de un aprendizaje emergente, producto de los acontecimientos experimentados en cada una de las sesiones. De igual manera, a través del trabajo grupal se genera un aprendizaje cooperativo en el que la relación entre alumnado y profesorado brinda un ambiente no solo motivador y gratificante, sino efectivo, en la medida en que está basado en la confianza y el respeto mutuos (Reid y Duke, 2015). Esta relación es promovida por un profesorado que no descuida el aspecto ético del proceso educativo (Elliot, 2014) y que es consciente de que la creación de una performance, tanto en el ámbito académico como, posteriormente, en el profesional, requiere de buenas relaciones interpersonales que optimicen el trabajo grupal y el resultado estético final (Thakar, 2015).

Así mismo, y respondiendo al primer objetivo de la investigación, se observa que algunos elementos de los procesos analizados pueden ser comprendidos desde una perspectiva performativa, utilizando herramientas provenientes de la EMP y del AMI, sin bien existen otros que podrían mejorarse. Es el caso de la utilización consciente del cuerpo y del espacio, tanto en formas convencionales como no convencionales, puesto que las experiencias performativas que diversifican el uso de estas herramientas corporales y espaciales resultarían de gran utilidad para el desarrollo profesional del estudiante de Música, desde el momento en que le hacen tomar conciencia de la importancia de su cuerpo en el espacio durante la realización de una performance, jugando estas categorías un papel decisivo tanto en el proceso como en el producto artístico final.

Por lo que se refiere a la motivación del alumnado (objetivo b), las entrevistas revelan una respuesta muy positiva respecto a la clase de combo, desde el momento en que les concede libertad para escoger el estilo musical a trabajar, de suerte que los jóvenes agradecen la flexibilidad del profesorado en cuanto a la interpretación instrumental individual y colectiva, así como al respeto por el criterio musical de cada cual, siempre dentro del marco de actuación establecido colectivamente. Por otra parte, el alumnado muestra una muy buena sintonía entre sí, estableciéndose relaciones interpersonales que se ven afianzadas por el propio hacer musical grupal a lo largo del tiempo.

En relación al tercer objetivo, se ha comprobado que las prácticas metodológicas llevadas a cabo promueven el empoderamiento del alumnado como futuro intérprete profesional, desde el momento en que las clases de combo pretenden asemejarse a la actividad real de un grupo musical profesional. Esto permite que aquél se familiarice con los procesos y las distintas situaciones que surgen con motivo de la creación grupal de una performance. De igual manera, se dota al discente con herramientas en aspectos como la dirección musical, las relaciones personales, los roles en el seno del ensemble o la toma de decisiones, entre otras; resultados positivos derivados de un aprendizaje cooperativo y que concuerdan con otros

@María Fernanda Ramírez Martínez y José A. Rodríguez-Quiles. The content of this article is the sole responsibility of the authors. The Revista Electrónica de LEEME and Universitat de València are not liable for any legal actions that may arise involving the article's content. Revista Electrónica de LEEME - Lista Electrónica Europea de Música en la Educación-. http://ojs.uv.es/index/php/LEEME/index ISSN: 1575-9563. Editores: Universidad de Valencia y Jesús Tejada. Visibilidad de esta revista: SCOPUS, Emerging Sources Citation Index (Clarivate), EBSCO, CINDOC (CSIC), Citefactor, COPAC, Dialnet, DICE (CSIC), DOAJ, e-revistas (CSIC), EBSCO Premier, ERIH+, Gale Cengage Learning, IN-RECS, IRESIE, LATINDEX, MIAR, OCLC Worldcat, RESH, REDIB, RILM Core Journals, SUDOC, ULRICHS. Esta revista es de acceso libre mediante licencia Creative Commons 4.0 CC by. Política de archivado: etiqueta verde SHERPA-ROMEO. 
estudios recientes en el ámbito de la formación del profesorado (Izquierdo, Martínez, Frutos y Moreno, 2019).

Dado que la investigación sugiere que el profesorado opta por plantear en sus clases un ambiente de aprendizaje flexible en torno a prácticas de carácter performativo e informal, valdría la pena formalizar dichas prácticas pedagógicas a fin de ofrecer a los docentes un corpus de conocimiento teórico-práctico sobre las mismas, todo ello a la luz de la EMP y del AMI (Woody, 2007; Green, 2008; Mantie, 2013; Rodríguez-Quiles, 2017, 2018). Desde este punto de vista, resultaría interesante la colaboración entre instituciones musicales, centros universitarios y grupos de investigación a fin de generar dinámicas educativas acordes con una educación postmoderna, también en el ámbito de la formación musical de carácter profesionalizado (Pulman, 2014; Mok, 2017).

\section{Agradecimientos}

Los autores agradecen tanto a la ESMUC como al profesorado y alumnado participantes en este estudio sus inestimables aportaciones.

\section{Referencias}

Abrahams, F., Rowland, M. y Kohler, K. (2012). Music Education behind Bars: Giving Voice to the Inmates and the Students Who Teach Them. Music Educators Journal, 98(4), 6773. doi: $10.1177 / 0027432112443711$

Austin, J.L. (2010). Cómo hacer cosas con palabras. Barcelona: Paidós.

Bachmann-Medik, D. (2006). Cultural Turns. Neuorientierungen in den Kulturwissenschaften. Reinbek bei Hamburg: Rowohlt.

Davidson, J.W. (2014). Introducing the Issue of Performativity. Music, Musicology Australia, 36(2), 179-188. doi: 10.1080/08145857.2014.958269

Deleuze, G. y Guattari, F. (2006). Mil mesetas. Capitalismo y esquizofrenia. Valencia: Pre Textos.

Duke, R. (2006). Beautiful Teaching. The American Music Teacher, 56(2), 22-24. Recuperado de: http://openmusiclibrary.org/article/16039/

Egger, J. (2019). Effects of cooperative learning on preservice elementary teachers' interest in and integration of music into core academic subjects. International Journal of Music Education, 37(4), 608-621. doi: 10.1177/0255761419852173

@María Fernanda Ramírez Martínez y José A. Rodríguez-Quiles. The content of this article is the sole responsibility of the authors. The Revista Electrónica de LEEME and Universitat de València are not liable for any legal actions that may arise involving the article's content. Revista Electrónica de LEEME - Lista Electrónica Europea de Música en la Educación-. http://ojs.uv.es/index/php/LEEME/index ISSN: 1575-9563. Editores: Universidad de Valencia y Jesús Tejada. Visibilidad de esta revista: SCOPUS, Emerging Sources Citation Index (Clarivate), EBSCO, CINDOC (CSIC), Citefactor, COPAC, Dialnet, DICE (CSIC), DOAI, e-revistas (CSIC), EBSCO Premier, ERIH+, Gale Cengage Learning, IN-RECS, IRESIE, LATINDEX, MIAR, OCLC Worldcat, RESH, REDIB, RILM Core Journals, SUDOC, ULRICHS. Esta revista es de acceso libre mediante licencia Creative Commons 4.0 CC by. Política de archivado: etiqueta verde SHERPA-ROMEO. 
Espinosa, I. de (2019). Innovando la docencia desde la formación del profesorado. Propuestas y realidades. Educatio Siglo XXI, 37(1), 251-254. Recuperado de: http://revistas.um.es/educatio/article/view/366121

Feichas, H. (2010). Bridging the gap: Informal learning practices as a pedagogy of integration. British Journal of Music Education, 27(1), 47-58. doi:10.1017/S0265051709990192

Fischer-Lichte, E. (2004). Ästhetik des Performativen. Frankfurt: Suhrkamp.

Fischer-Lichte, E. (2012). Performativität. Eine Einführung. Bielefeld: transcript Verlag.

Flores, S. (2007). Principales acercamientos al uso de la música popular actual en la Educación Secundaria. Revista Electrónica LEEME, 19, 1-16. Recuperado de: http://ojs.uv.es/index.php/LEEME/article/9763/9197

Green, L.P. (2002). How popular musicians learn: A way ahead for music education. Abingdon, GB: Ashgate.

Green, L. P. (2004). What can Music Educators learn from Popular Musicians? En C.X. Rodríguez (Ed.), Bridging the Gap. Popular Music and Music Education (pp.224-241). Reston, VA: MENC: The National Association for Music Education.

Green, L.P. (2005). The music curriculum as lived experience: children's 'natural' music learning processes. Music Educators' Journal, 91(4), 37-42. doi: 10.2307/3400155

Green, L.P. (2006). Popular music education in and for it self, and for 'other' music: current research in the classroom. International Journal of Music Education, 24(2), 101-118. doi: $10.1177 / 0255761406065471$

Green, L.P. (2008). Music, informal learning and the school: A new classroom pedagogy. Abingdon, GB: Ashgate.

Hall, R. (2014). Enhancing the Popular Music Ensemble. Workshop and maximising student potential through the integration of creativity. International Journal of MusicEducation, 33(1), 103-112, doi: 10.1177/0255761414533310

Hargreaves, D. (2012). Musical imagination: Perception and production, beauty and creativity. Psychology of Music, 40(5), 539-557. doi: 10.1177/0305735612444893

Harrison, S., Lebler, D., Carey, G., Hitchcock, M. y O’Bryan, J. (2013). Making music or gaining grades? Assessment practices in tertiary music ensembles. British Journal of Music Education, 30(1), 27-42. doi: 10.1017/S0265051712000253

@María Fernanda Ramírez Martínez y José A. Rodríguez-Quiles. The content of this article is the sole responsibility of the authors. The Revista Electrónica de LEEME and Universitat de València are not liable for any legal actions that may arise involving the article's content. Revista Electrónica de LEEME - Lista Electrónica Europea de Música en la Educación-. http://ojs.uv.es/index/php/LEEME/index ISSN: 1575-9563. Editores: Universidad de Valencia y Jesús Tejada. Visibilidad de esta revista: SCOPUS, Emerging Sources Citation Index (Clarivate), EBSCO, CINDOC (CSIC), Citefactor, COPAC, Dialnet, DICE (CSIC), DOAJ, e-revistas (CSIC), EBSCO Premier, ERIH+, Gale Cengage Learning, IN-RECS, IRESIE, LATINDEX, MIAR, OCLC Worldcat, RESH, REDIB, RILM Core Journals, SUDOC, ULRICHS. Esta revista es de acceso libre mediante licencia Creative Commons 4.0 CC by. Política de archivado: etiqueta verde SHERPA-ROMEO. 
Izquierdo, T., Martínez, E.A., Frutos, A.E. y Moreno, J.R. (2019). El aprendizaje cooperativo en la formación de maestros de Educación Primaria. Revista de Investigación Educativa, 37(2), 543-559. doi: 10.6018/rie.37.2.369731

Jenkins, P. (2011). Formal And Informal Music Educational Practices. Philosophy of Music Education Review, 19(2), 179-197. doi: 10.2979/philmusieducrevi.19.2.179

Karlsen, S. (2010). BoomTown music education and the need for authenticity - informal learning put into practice in swedish post-compulsory music education. British Journal of Music Education, 27(1), 35-46. doi: 10.1017/S0265051709990180

Kartomi, M. (2014). Concepts, Terminology and Methodology in MusicPerformativity Research. Musicology Australia, 36(2), 189-208. doi: 10.1080/08145857.2014.958268

Locke, K. (2008). Music Education and Ethical Judgment in the Postmodern Condition. Action, Criticism, and Theory for Music Education, 7(1), 74-87. Recuperado de: http://act.maydaygroup.org/articles/Locke7 1.pdf

Mantie, R. (2013). A comparison of "popular music pedagogy" discourses. Journal of Research in Music Education, 61(3), 334-352. doi: 10.1177/0022429413497235

Mok, A.O. (2017). Informal learning: A lived experience in a university musicianship class. British Journal of Music Education, 34(2), 169-188. doi: 10.1017/S0265051716000498

Mok, A.O. (2018). Formal or informal - which learning approach do music majors prefer? International Journal of Music Education, 36(3), 380-393. doi: $10.1177 / 0255761418761258$

Nichols, J. (2016). Sharing the Stage: Ethical Dimensions of Narrative Inquiry in Music Education. Journal of Research in Music Education, 63(4), 439-454. doi: $10.1177 / 0022429415617745$

Pineau, E.L. (2005). Teaching Is Performance: Reconceptualizing a Problematic Metaphor. En B.K. Alexander (Ed.), Performances Theory In Education. Power, Pedagogy and the Politics of Identity (pp.15-39). New York: Routledge.

Pulman, M. (2014). Popular music pedagogy: Band rehearsals at British universities. International Journal of Music Education, 32(3), 296-310. doi: $10.1177 / 0255761413491207$

Reid, A. y Duke, M. (2015). Student for student: Peer learning in music higher education. International Journal of Music Education, 33(2), 222-232. doi: $10.1177 / 0255761415569107$

@María Fernanda Ramírez Martínez y José A. Rodríguez-Quiles. The content of this article is the sole responsibility of the authors. The Revista Electrónica de LEEME and Universitat de València are not liable for any legal actions that may arise involving the article's content. Revista Electrónica de LEEME - Lista Electrónica Europea de Música en la Educación-. http://ojs.uv.es/index/php/LEEME/index ISSN: 1575-9563. Editores: Universidad de Valencia y Jesús Tejada. Visibilidad de esta revista: SCOPUS, Emerging Sources Citation Index (Clarivate), EBSCO, CINDOC (CSIC), Citefactor, COPAC, Dialnet, DICE (CSIC), DOAJ, e-revistas (CSIC), EBSCO Premier, ERIH+, Gale Cengage Learning, IN-RECS, IRESIE, LATINDEX, MIAR, OCLC Worldcat, RESH, REDIB, RILM Core Journals, SUDOC, ULRICHS. Esta revista es de acceso libre mediante licencia Creative Commons 4.0 CC by. Política de archivado: etiqueta verde SHERPA-ROMEO. 
Richerme, L. (2017). A feminine and poststructural extension of cosmopolitan ethics in music education. International Journal of Music Education, 35(3), 414-424. doi: $10.1177 / 0255761416667470$

Rodríguez-Quiles, J.A. (2017). Rethinking Music Education: Towards a Performative Turn.

En J.A. Rodríguez-Quiles (Ed.), Internationale Perspektiven zur Musik(lehrer)ausbildung in Europa (pp.19-37). Potsdam: UP Verlag.

Rodríguez-Quiles, J.A. (2018). La música como rizoma. Bases para una educación musical performativa. Revista Musical Chilena, 72(229), 139-150. doi:10.4067/s071627902018000100139

Stake, R.E. (1998). Investigación con estudio de casos. Madrid: Ediciones Morata.

Thakar, M. (2015). Looking for the "Harp" Quartet: An Investigation into Musical Beauty. Rochester: University of Rochester Press.

Tobias, E.S. (2013). Composing, song writting, and producing: Informing popular music pedagogy. Researh Studies in Music Education, 35(2), 213-237. doi: $10.1177 / 1321103 X 13487466$

Vidmar, T. (2014). Selected Aspects of Non-Formal Education in Ancient Greece, Middle Ages and the Reformation. En I.Ž. Žagar y P. Kelava (Eds.) (2014). From formal to nonformal: Education, learning and knowledge [Versión epub]. Recuperado de: http://www.researchgate.net/publication/325107863_From_Formal_to_Non-

Formal Education Learning and Knowledge

Volbers, J. (2014). Performative Kultur. Wiesbaden: Springer.

Whitener, J. (2016). Using the elements of cooperative learning in school band classes in the United States. International Journal of Music Education, 34(2), 219-233. doi: $10.1177 / 0255761414541865$

Woody, R.H. (2007). Popular Music in the School: Remixing the issues. Music Educators' Journal, 93(4), 32-37. doi: 10.1177/002743210709300415

@María Fernanda Ramírez Martínez y José A. Rodríguez-Quiles. The content of this article is the sole responsibility of the authors. The Revista Electrónica de LEEME and Universitat de València are not liable for any legal actions that may arise involving the article's content. Revista Electrónica de LEEME - Lista Electrónica Europea de Música en la Educación-. http://ojs.uv.es/index/php/LEEME/index ISSN: 1575-9563. Editores: Universidad de Valencia y Jesús Tejada. Visibilidad de esta revista: SCOPUS, Emerging Sources Citation Index (Clarivate), EBSCO, CINDOC (CSIC), Citefactor, COPAC, Dialnet, DICE (CSIC), DOAJ, e-revistas (CSIC), EBSCO Premier, ERIH+, Gale Cengage Learning, IN-RECS, IRESIE, LATINDEX, MIAR, OCLC Worldcat, RESH, REDIB, RILM Core Journals, SUDOC, ULRICHS. Esta revista es de acceso libre mediante licencia Creative Commons 4.0 CC by. Política de archivado: etiqueta verde SHERPA-ROMEO. 